\title{
Rheological behavior of colloidal suspension with long-range interactions
}

\author{
S. Arietaleaniz \\ KIRO GRIFOLS S.L. Polo de Innovación Garaia, Goiru Kalea 1, Edificio B, Planta 2, 20500 Arrasate, Spain \\ and Departamento de Ingeniera Biomédica (TECNUN), Universidad de Navarra, 20009 San Sebastian, Spain \\ P. Malgaretti* \\ Max-Planck-Institut für Intelligente Systeme, Heisenbergstr. 3, 70569 Stuttgart, Germany \\ and IV. Institut für Theoretische Physik, Universität Stuttgart, Pfaffenwaldring 57, 70569 Stuttgart, Germany
}

I. Pagonabarraga

Departament de Física de la Materia Condensada and Institute of Complex Systems (UBICS), Universitat de Barcelona, Barcelona 08028, Spain

and CECAM Centre Européen de Calcul Atomique et Moléculaire, École Polytechnique Fédérale de Lausanne (EPFL), Lausanne CH-1015, Switzerland

R. C. Hidalgo

Departamento de Física y Matemática Aplicada. Facultad de Ciencias, Universidad de Navarra, 31080 Pamplona, Spain

(Received 5 May 2018; published 10 October 2018)

\begin{abstract}
In this work, we study the constitutive behavior of interacting colloidal suspensions for moderate and high concentrations. Specifically, using a lattice Boltzmann solver, we numerically examine suspensions flowing through narrow channels, and explore the significance of the interaction potential strength on the system's macroscopic response. When only a short-range interaction potential is considered, a Newtonian behavior is always recovered and the system's effective viscosity mostly depends on the suspension concentration. However, when using a Lennard-Jones potential we identify two rheological responses depending on the interaction strength, the volume fraction, and the pressure drop. Exploiting a model proposed in the literature we rationalize the simulation data and propose scaling relations to identify the relevant energy scales involved in these transport processes. Moreover, we find that the spatial distribution of colloids in layers parallel to the flow direction does not correlate with changes in the system macroscopic response; but, interestingly, the rheology changes do correlate with the spatial distribution of colloids within individual layers. Namely, suspensions characterized by a Newtonian response display a cubiclike structure of the colloids within individual layers, whereas for suspensions with non-Newtonian response colloids organize in a hexagonal structure.
\end{abstract}

DOI: 10.1103/PhysRevE.98.042603

\section{INTRODUCTION}

Transport of particles in confined geometries appears in many technological and biological processes [1,2]. Examples span from engineering applications such as the injection of fuel in engines, energy harvesting devices [3] and microfluidics [4] to physiological processes such as blood flow through vessels [5-8] and ionic pumping transport in channels [9]. Interestingly, the rheology of all these systems is quite complex when compared to that of Newtonian fluids due to the stresses induced by the interactions among suspended particles. Accordingly, these systems are usually grouped under the lemma of soft glassy materials that includes concentrated emulsions, foams, colloids, and even macroscopic granular assemblies $[1,2]$.

In the last decade, a number of experimental techniques were introduced, allowing us to capture the rheology of soft

\footnotetext{
*malgaretti@is.mpg.de

†raulcruz@unav.es
}

glassy materials such as $\mathrm{x}$-ray tomography [10-12], dynamic light scattering [13], magnetic resonance imaging [14,15], and high-frequency ultrasonic speckle velocimetry [16]. In particular, previous outcomes [13-22] demonstrate that the rheological response of colloidal suspensions is very sensitive to the confining conditions, as well as, strongly depending on the system composition. On one hand, for low volume fractions of the dispersed phase the Newtonian response is generally recovered. On the contrary, upon increasing volume fraction the interactions among particles begin to play a role. As a result, particles rearrangements occur, which leads to nonlinear macroscopic response and enhancing of the yield stress. Several research groups have proposed to rationalize these complex response, assuming that the macroscopic flow is produced by a succession of local elastic deformations and irreversible plastic rearrangements [23-31]. Thus, these events cause long-range stress fluctuations over the system, which create localized fragile zones where the system flows. Moreover, correlations between those plastic events have been detected [32]. In particular, Goyon et al. [14,15] characterized 
the rheology of soft glassy materials confined between parallel plates by introducing a nonuniform effective field named nonlocal fluidity.

In this paper, we go one step further exploring the flow of soft glassy materials in a confined geometry. We numerically examine three-dimensional (3D) suspensions of colloids flowing through narrow channels at low Reynolds number, focusing on the relevance of the interaction potential among suspended colloids on the overall rheology of the system. Our results show that the interactions among colloids strongly affect the rheology of the system and lead to a non-Newtonian response even at smaller volume fractions, compared to systems of noninteracting colloids. Interestingly, we find that the onset of the non-Newtonian response is controlled by the ratio between the magnitude of the applied pressure drop and the magnitude of the colloid-colloid interaction potential. When the attractive interaction potential is strong, for low values of the pressure drop the system shows a non-Newtonian response whereas upon increasing the pressure drop it eventually displays a Newtonian behavior. Finally, in order to capture the velocity profile in the non-Newtonian regime we exploit a previous mentioned model $[14,15]$ that account for inhomogeneous transport coefficients of the fluid. It is worth mentioning that a similar effort was carried out in two dimensions, exploring the rheological response of droplets [33].

\section{COLLOIDAL SUSPENSION FLOWING THROUGH A NARROW CHANNEL (LATTICE BOLTZMANN SCHEME)}

The system under study is composed by $N$ colloidal particles of radius $R$, suspended in a Newtonian fluid (see Fig. 1). The system is confined by solid walls along the $z$ direction and periodic boundary conditions are applied along $x$ and $y$. A lattice Boltzmann (LB) approach is used to model the fluid. This method recovers the solution of the Navier-Stokes equations, and it has provided significant results examining the rheological response of complex liquids [34-39].

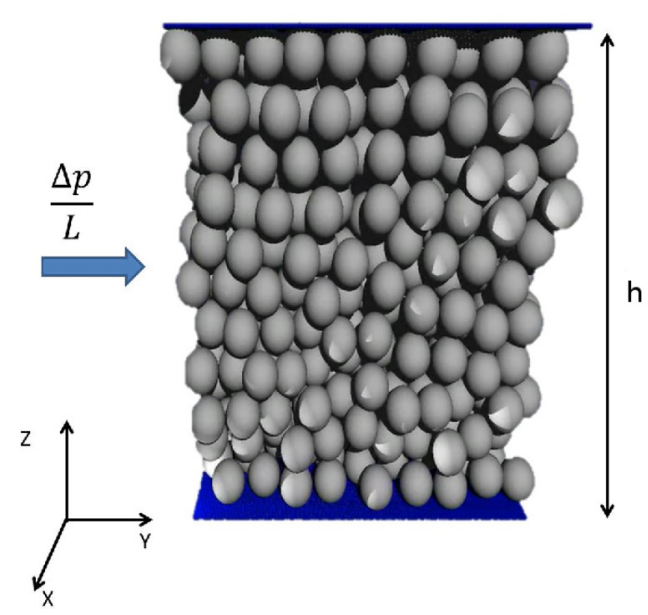

FIG. 1. Illustration of the colloidal suspension, which moves driven by a pressure drop $\frac{\Delta p}{L}$ acting on the $Y$ direction. The system is confined in the $Z$ direction by solid walls and periodic boundaries condition are imposed on $X$ and $Y$ directions.
According to the LB scheme the fluid dynamics is read out from the single-particle distribution function $f(\vec{r}, t)$ [35]. Hence, at each lattice node, the discretized distribution function $f_{i}(\vec{r}, t)$ evolves at discrete time steps $\Delta t$ as,

$$
f_{i}\left(\vec{r}+\vec{c}_{i} \Delta t ; t+\Delta t\right)=f_{i}(\vec{r} ; t)+\Omega_{i j}\left(f_{j}^{e q}(\vec{r} ; t)-f_{j}(\vec{r} ; t)\right) .
$$

Note, this evolution rule accounts for the linear momentum streaming to the neighbors nodes $j$ due to the liquid advection motion of velocity $\vec{c}_{i}$. Moreover, a collision operator $\Omega_{i j}$ rules the relaxation process toward an equilibrium state, characterized by $f_{j}^{e q}(\vec{r} ; t)$. In computing, we use LUDWIG $[35,40]$ a lattice Boltzmann implementation, which is able to reproduce the behavior of complex fluids, and include a multirelaxation collision operator $\Omega_{i j}$ [40]. The system geometry is a 3D cubic lattice with 19 allowed velocities $\vec{c}_{i}$ known as $D_{3} Q_{19}$ scheme [35]. We use units such that the mass of the nodes, the lattice spacing, and the time step are one and the kinematic viscosity is $v$. Thermal fluctuations $k_{B} T \neq 0$ are incorporated in the lattice Boltzmann model adding an additional noise term $\xi_{i}\left(k_{B} T\right)$ in Eq. (1) [41]. This additional contribution introduces fluctuations in the populations in each phase-space cell. The stochastic properties of this term are chosen to ensure that the fluctuation dissipation theorem in equilibrium is satisfied [41,42].

As mentioned above, the system is confined by solid walls on the $z$ direction. To compare with experimental conditions, appropriate boundary conditions at the walls are needed $[40,43]$. In LUDWIG walls are implemented by applying socalled stick boundary conditions [40]. Thus, during propagation, the component of the distribution function that would propagate into the wall node is bounced back and ends up back at the fluid node but pointing in the opposite direction. This procedure produces stick boundary conditions at the middle point of the vector joining the wall and fluid nodes.

The rheological response of the colloidal suspensions is realistically modeled, assuming that the solid particles interact with the surrounding fluid also through bounce-back on the links. Hence, the total force and torque acting on the colloid are determined using the mechanical constraint that the momentum exchange between the solid particle and the nodes from bounce-back vanishes. The interaction between the colloids is defined by two interaction potentials. A shortterm soft potential that reads as,

$$
v^{S P}= \begin{cases}\epsilon_{S P}\left(\frac{\sigma_{S P}}{r}\right)^{v_{o}} & r<R+r_{c} \\ 0 & \text { else }\end{cases}
$$

where $\epsilon_{S P}$ accounts for the strength of the contact interaction, $\sigma$ is the length scale and $\nu_{0}$ characterize the range of the particle-particle interaction. The numerical implementation of the interaction potential Eq. (2) ensures colloids do not overlap at the hard-core radius $R$ when introducing a socalled hydrodynamic radius $R_{h}$ [44]. In general, simulating multiparticle suspension all distance calculations are based on the hydrodynamic radius $R_{h}$, and to obtain accurate results it is then essential to use a calibrated value [45], which is typically larger than the physical radius $R$. Besides this, $v^{S P}$ is truncated at a cutoff distance of $r_{c}=0.25$ (in lattice units). Additionally, a long range of interaction Lennard-Jones 
potential is also used,

$$
v^{L J}=4 \epsilon_{L J}\left(\left(\frac{\sigma_{L J}}{r}\right)^{12}-\left(\frac{\sigma_{L J}}{r}\right)^{6}\right),
$$

where $\epsilon_{L P}$ accounts for the strength of the contact interaction. Accordingly, a pair of forces $\vec{F}_{i j}=-\vec{F}_{j i}$ with equal magnitudes $\left|\nabla v^{L J}\right|$, is applied to interacting colloids at a distance $r$, but $v^{L J}$ is truncated at a cutoff distance of $r_{c}=5 R$. In all cases presented here the values of $\sigma_{S P}=1.0, v_{0}=1.0, \sigma_{L J}=$ $2 R_{h}, R=5.0$, and $R_{h}=5.2$ (in lattice units) are fixed. Note that using those parameter values the interaction potentials Eq. (2) and Eq. (3) are mainly locally repulsive and long-range attractive, respectively.

In the simulations $N=1000$ colloidal particles are initially distributed in random positions inside the simulation box whose dimensions along $x$ and $y\left(w=w_{x}=w_{y}\right)$ are adjusted in order to attain the desired volume fraction, $\phi \in$ $[0.30,0.52]$, whereas for the confining direction we fixed $h=104$ nodes. Fluid parameters are chosen to ensure a low Reynolds number $\operatorname{Re}=V_{m} h / v$ where $v$ is the fluid kinematic viscosity and $V_{m}$ is the maximum velocity, which is measured at the center of the channel. The fluid is subject to a pressure gradient along the $y$ direction, $\frac{\Delta p}{L}$, which results in a uniform force parallel to the walls, mimicking a pressure driven flow that pushes the colloidal particles through the channel. The force induces the flow of the suspension, which reaches a steady state after a short transient. In the following, all the values of pressure gradient $\frac{\Delta p}{L}$ are in lattice units $\left(\rho k_{B} T / l\right.$ being $l$ the lattice unit and $\rho$ the number density of the fluid). In the following we consider a pressure gradient $\frac{\Delta p}{L}=5 \times$ $10^{-5} \rho \frac{k_{B} T}{l}$ that corresponds to a pressure gradient of $\frac{\Delta p}{L} \simeq$ $6 \times 10^{3} \mathrm{~Pa} / \mathrm{m}$. We remark that this value of the pressure drop is well within the typical experimental range (see Ref. [46]).

\section{NONUNIFORM RHEOLOGICAL FORMULATION}

In general, the rheological response of a liquid with negligible yield stress reads as,

$$
\sigma(z)=\mu(\dot{\gamma}(z)) \dot{\gamma}(z),
$$

where the shear strain $\dot{\gamma}(z)$ is defined as

$$
\dot{\gamma}=\frac{\partial v_{y}(z)}{\partial z}
$$

and $\mu(\dot{\gamma}(z))$ is the effective viscosity.

Goyon et al. $[14,15]$ examined the rheological behavior of complex flows introducing a novel nonuniform flow rule. According to Refs. [14,15], the fluidity $f(z)$ is defined as the ratio between shear rate and shear stress

$$
f(z)=\frac{\dot{\gamma}(z)}{\sigma(z)}=\frac{1}{\mu(\dot{\gamma}(z))}
$$

having the dimension of inverse viscosity. Moreover, the model postulates that the fluidity $f(z)$ is the solution of:

$$
f(z)=f_{\text {bulk }}+\xi^{2} \frac{\partial^{2} f(z)}{\partial^{2} z}
$$

and it depends parametrically on $\xi$ (the so-called fluidity length) and $f_{\text {bulk }}$. We remark that in our case the limit $\xi \rightarrow 0$ corresponds to a Newtonian fluid. Here, we study numerically a pressure driven flow in a rectangular channel with height $h$. In that geometry, the shear stress varies spatially according to

$$
\sigma(z)=-\frac{\Delta p}{L} z
$$

where $\frac{\Delta p}{L}$ is the constant pressure gradient along the channel. Note that two boundary conditions are needed to obtain the solution of the fluidity, Eq. (7) and one boundary condition for the velocity profiles $v_{y}(z)$. Using the numerical data, we deduce the wall fluidity estimating the velocity gradient at the wall $\dot{\gamma}( \pm h / 2)=\dot{\gamma}_{w}$, finding $f(-h / 2)=f(h / 2)=f_{w}=$ $\frac{\sigma( \pm h / 2)}{\dot{\gamma}_{w}}$. Thus, using Eq. (8) the solution of Eq. (7) reads

$$
f(z)=\frac{f_{w}-f_{\text {bulk }}}{\cosh \left(\frac{h}{2 \xi}\right)} \cosh \left(\frac{z}{\xi}\right)+f_{\text {bulk }} .
$$

Finally the velocity profiles $v_{y}(z)$ can be analytically deduced form Eq. (4), using the momentum balance Eq. (8), and obtaining

$$
\frac{d v_{y}(z)}{d z}=\frac{\Delta p}{L} z\left(\frac{f_{w}-f_{\text {bulk }}}{\cosh \left(\frac{h}{2 \xi}\right)} \cosh \left(\frac{z}{\xi}\right)+f_{\text {bulk }}\right) .
$$

Each particular solution of Eq. (10) involves an integration constant that we fix by imposing that the velocity at the center of the channel $V_{m}=v_{y}(0)$ matches the one obtained from the numerical simulations. We remark that following this procedure the velocity profiles within the channel $v_{y}(z)$ is totally determined by the values of fluidity length $\xi$ and bulk viscosity $f_{\text {bulk }}$, which will be used as fitting parameters when comparing the LB numerical results with Eqs. (5)-(9).

\section{RESULTS AND DISCUSSION}

In the simulations, the colloidal suspension is subject to a constant pressure gradient $\frac{\Delta p}{L}$, which induces the flow. In general, we obtain that the suspension yield stress is practically zero and after a short time, a steady state is reached.

First, we characterize the rheology of the system in the absence of long-range interactions $\left(\epsilon_{L J}=0\right)$. For such a regime, the system shows a Newtonian response regardless the strength of the short-range interaction $\epsilon_{S P}$. As shown in Fig. 2, the velocity profiles obtained for different volume fractions all collapse on a parabola, i.e., the system behaves as a Newtonian fluid. Interestingly, the data indicate that the effective viscosity $\eta_{\text {eff }}=\frac{h^{2}}{8 V_{m}} \frac{\Delta p}{L}$ increases monotonically with the volume fraction $\phi$. As expected, for small values of $\phi$ the dependence of $\eta_{\text {eff }}(\phi) / \eta_{o}$ is compatible with Batchelor's formula [47] whereas for larger values of $\phi$ it is better captured by [48]

$$
\eta_{\mathrm{eff}}=\eta_{0}\left[1-\frac{\phi}{1-c \phi}\right]^{-\frac{5}{2}},
$$

where $c=\frac{1-\phi_{c}}{\phi_{c}}$ and $\phi_{c} \simeq 0.74$, which is the maximum packing fraction of hard spheres in three dimensions. For comparison, the expressions proposed by Quemada [49] and Krieger [50] are also included. Our results show the colloids are not homogeneously distributed across the channel, rather they accumulate at specific locations [see the points location in Fig. 2(a)]. Indeed, for the largest value of $\phi$ that we explore, 

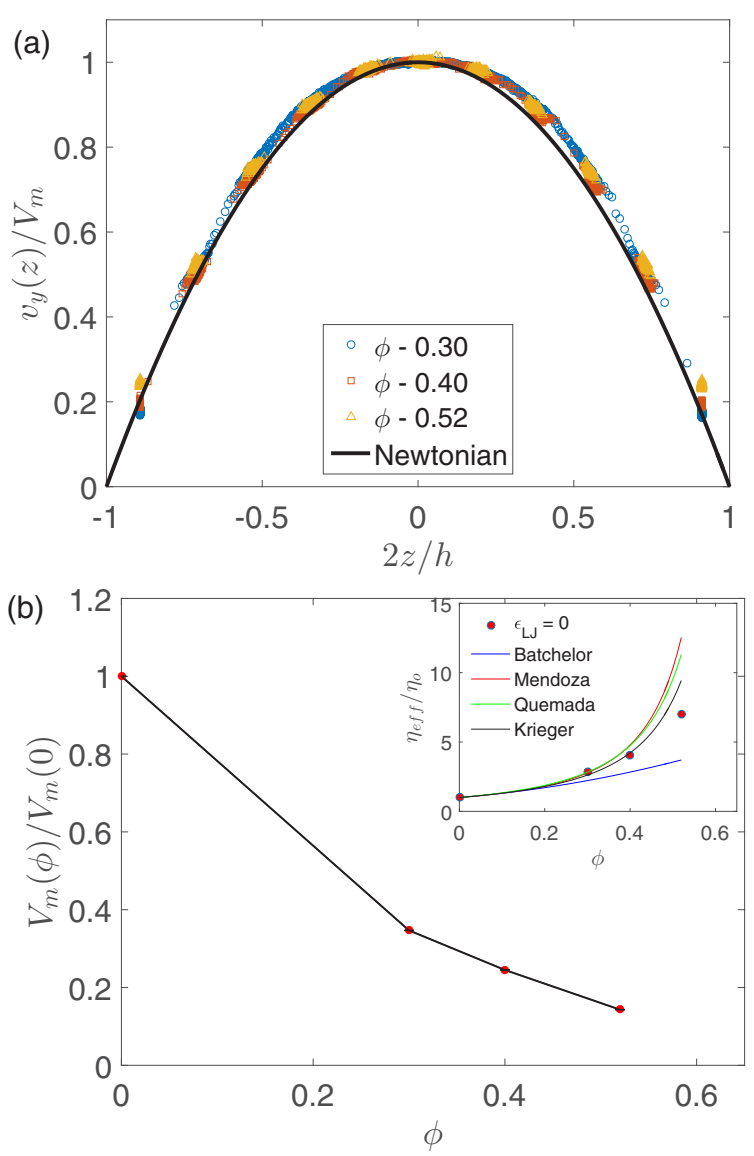

FIG. 2. (a) Velocity profiles obtained numerically. The data correspond to colloidal suspensions without long-range interaction $\left(\epsilon_{S P}=0.5\right.$ and $\left.\epsilon_{L J}=0\right), \frac{\Delta p}{L}=5 \times 10^{-5}, \epsilon_{S D}=0.5$. Outcomes for several volume fractions $\phi$ are shown. The velocity values are rescaled with the maximum velocity $V_{m}$ and compared with a typical Newtonian response. (b) The maximum velocity $V_{m}(\phi)$ as a function of $\phi$ is illustrated, the values are rescaled with $V_{m}(0)=\frac{h^{2}}{8 \eta_{0}} \frac{\Delta p}{L}$. In the inset the values of effective viscosity $\eta_{\text {eff }} / \eta_{o}$ are compatible with the Batchelor's analytic prediction for non-Brownian suspension. $\eta_{o}$ is the viscosity of the liquid, i.e., $\eta_{o}=\eta_{\text {eff }}(0)$.

the interparticle distance along the $z$ direction is smaller than the particles diameter $2 R$, suggesting that the particles are spatially distributed in a honeycomblike structure. Such an observation justifies the value of $\phi_{c}=0.74$ used in Eq. (11). We argue the system linear response is expected because the volume fraction $\phi$ is still low. We remark that non-Newtonian response was found experimentally for systems with $\phi>0.62$ $[14,15]$.

Next, we turn on the long-range Lennard-Jones interaction potential with strength $\epsilon_{L J}$ [see Eq. (3)]. Figure 3 summarizes a systematic study, exploring the macroscopic response of the system varying $\epsilon_{L J}$, while keeping constant the pressure gradient $\frac{\Delta p}{L}=5 \times 10^{-5}$ and the volume fraction $\phi=0.52$. Interestingly, the strength of long-range interaction potential $\epsilon_{L J}$ has a significant impact on the macroscopic rheological response, as shown in Fig. 3(a), or alternatively in Fig. 3(b). In contrast, the maximum velocity is not sensitive to the magnitude of the short-range interaction $\epsilon_{S D}$ [see insets of Fig. 3(a) and Fig. 3(b)]. In particular, Fig. 3(a) shows that
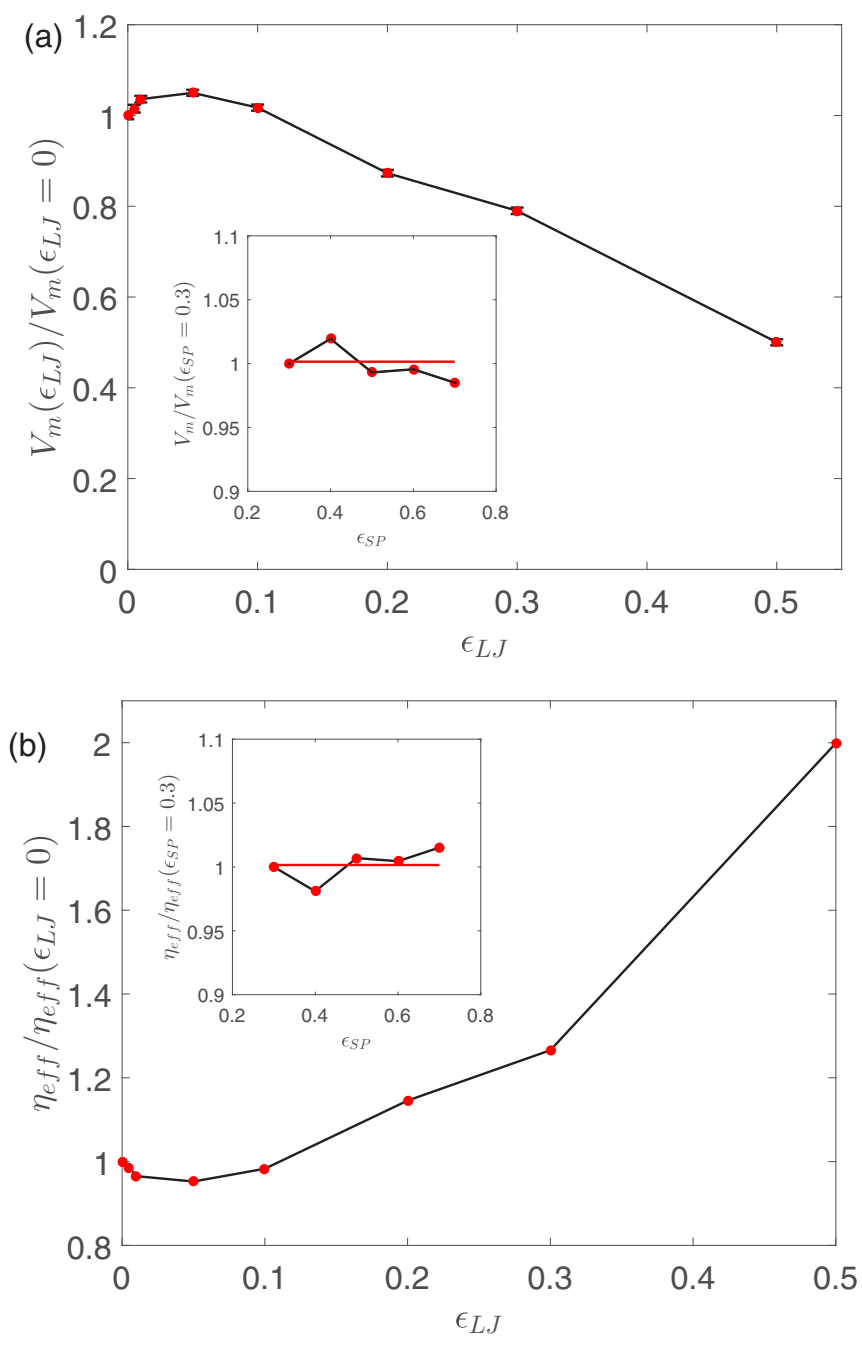

FIG. 3. Maximum velocity $V_{m}$ (a) in the channel as a function on $\epsilon_{L J}$, keeping $\epsilon_{S D}=0.5$, the values are rescaled with the maximum velocity obtained for $\epsilon_{L J}=0$. (b) The corresponding effective viscosity $\eta_{\text {eff }}$, the values are rescaled with $\eta_{\text {eff }}\left(\epsilon_{L J}=0\right)$. The insets illustrates the same but as a function of $\epsilon_{S D}$ keeping $\epsilon_{L J}=0$.

the maximum velocity decreases and effective viscosity [see Fig. 3(b)] grows upon increasing $\epsilon_{L J}$. More interesting, a nonuniform rheological response is typically found when $\epsilon_{L J} \neq 0$.

In order to rationalize the simulations data, we use the model presented in Sec. III. Figure 4(a) illustrates the velocity profiles obtained numerically, for different values of $\epsilon_{L J}$ at constant volume fraction $\phi=0.52$ and pressure gradient $\frac{\Delta p}{L}=5 \times 10^{-5}$. For comparison, in each case the analytic solution of Eq. (10) is also included. Interestingly, upon increasing $\epsilon_{L J}$ the velocity profiles depart from the Poiseuillelike profile typical of Newtonian fluids and a plateau sets at the center of the channel. Moreover, it correlates with the increasing of the shear rate close to the walls. We fit the velocity profiles using Eqs. (5)-(9), while $f_{\text {bulk }}$ and $\xi$ are fitting parameters. Figure 4(b) shows the bulk fluidity profiles $f(z)$, i.e., Eq. (9), normalized by $f_{\text {bulk }}^{*}(\phi)=\frac{1}{\eta_{\text {eff }}(\phi)}$ where $\eta_{\text {eff }}(\phi)$ is calculated using Eq. (11). Interestingly, Fig. 4(b) shows that for larger values of $\epsilon_{L J}$ the value of the fluidity 

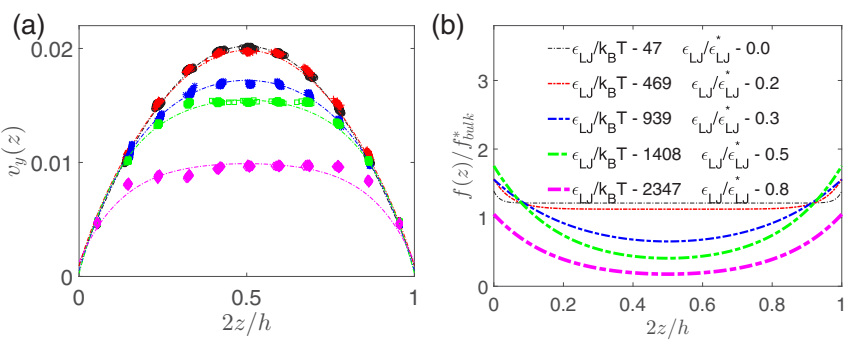

FIG. 4. System response obtained varying the strengths of longrange interaction $\epsilon_{L J}$, at constant $\phi=0.52$ and $\frac{\Delta p}{L}=5 \times 10^{-5}$. (a) Velocity profiles numerical (symbols) compared with the analytic predictions (lines) solution Eq. (10), see (b) for the color legend. In (b) the corresponding fluidity $f(z)$ profiles Eq. (7) are shown.

is quite reduced and its profile becomes nonuniform all along the channel transverse direction. The dependence of the fitting parameters, namely $f_{\text {bulk }}$ and $\xi$ on $\epsilon_{L J}$ is referred to further in the text.

Exploring the dependence of the onset of non-Newtonian regime on the suspension volume fraction $\phi$, we perform simulations varying $\phi$, for $\epsilon_{L J} \neq 0$. Figure 5(a) shows the velocity profiles obtained numerically for different values of $\phi$, keeping constant the strength of Lennard-Jones potential $\epsilon_{L J}=0.2$ and the pressure gradient $\frac{\Delta p}{L}=5 \times 10^{-5}$. For comparison, in each case the analytic solution of Eq. (10) is also included. As expected, the velocity profiles [see Fig. 4(a)] deviate from the Newtonian regime upon increasing the volume fraction $\phi$. The corresponding fluidity profiles [see Fig. 4(b)] are nonuniform along the transverse section of the channel, highlighting the onset of the non-Newtonian regime. Interestingly, when $\epsilon_{L J} \neq 0$ the non-Newtonian response is detected for volume fractions notably lower than $\phi=0.62$, which is the critical value obtained experimentally, in the absence of long-ranged interactions $[14,15]$.

Complementarily, we simulate cases varying the pressure drop $\frac{\Delta p}{L}$ at constant $\phi=0.52$ and $\epsilon_{L J}=0.2$. The velocity and fluidity profiles are presented in Fig. 6(a) and Fig. 6(b), respectively. Interestingly, the non-Newtonian response is attained for smaller values of $\frac{\Delta p}{L}$, suggesting that in that regime the particle-particle interaction potential is dominant. Whereas the linear response is recovered for large values
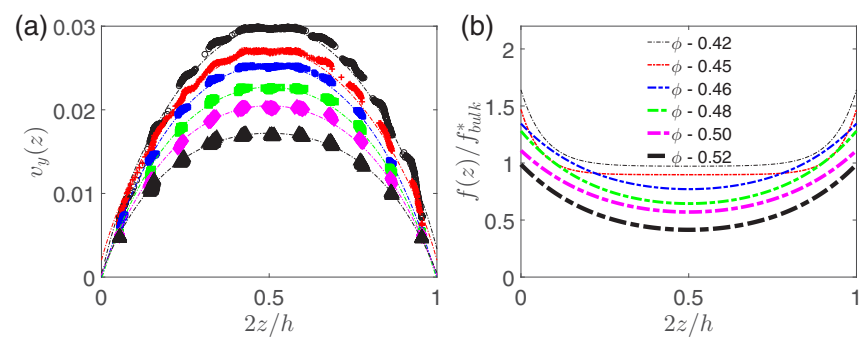

FIG. 5. System response obtained varying the colloidal volume fractions $\phi$, at constant $\epsilon_{L J}=0.2$ and $\frac{\Delta p}{L}=5 \times 10^{-5}$. (a) Velocity profiles numerical (symbols) compared with the analytic predictions (lines) solution of Eq. (10), see (b) for the color legend. In (b) the corresponding fluidity $f(z)$ profiles Eq. (7) are shown.
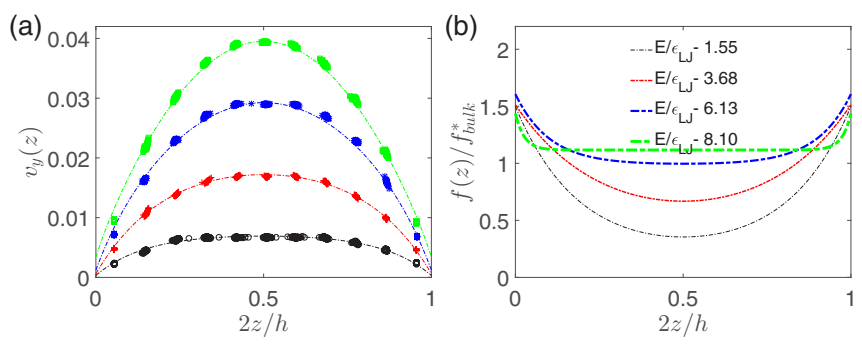

FIG. 6. System response obtained varying the pressure drop $\frac{\Delta p}{L}$ at constant $\phi=0.52$ and $\epsilon_{L J}=0.2$. (a) Velocity profiles numerical (symbols) compared with the analytic predictions (lines) solution Eq. (10), see (b) for the color legend. In (b) the corresponding fluidity $f(z)$ profiles Eq. (7) are shown.

of $\frac{\Delta p}{L}$, where the liquid-mediated hydrodynamic interactions play the most significant role.

\section{A. Onset of the nonuniform rheological behavior}

Next, we identify the onset of nonuniform rheological response, as well as the interrelationship between the external forcing, $\frac{\Delta p}{L}$, Lennard-Jones potential, $\epsilon_{L J}$, and volume fraction, $\phi$. As a first step, we clarify the significance of the three energy scales of the system, namely the thermal energy, $k_{B} T$, the long-ranged interaction strength $\epsilon_{L J}$, and the energy, $E$, associated to the viscous forces. Indeed the latter can be easily expressed via its time derivative, namely the dissipated power:

$$
\dot{E}=f_{d}\langle V\rangle \times\langle V\rangle=6 \pi \eta_{l} R\langle V\rangle^{2},
$$

where $\eta_{l}$ is viscosity of the fluid phase $\eta_{l}=\eta_{0}$ and $\langle V\rangle$ is the mean velocity of the fluid, which can be estimated in terms of the effective viscosity of the suspension $\eta_{\text {eff }}(\phi)$, and reads as

$$
\langle V\rangle=\frac{2}{3}\left(\frac{h^{2}}{8 \eta_{\mathrm{eff}}(\phi)} \frac{\Delta p}{L}\right),
$$

the average speed of a Newtonian fluid in a rectangular channel. In order to obtain an energy scale, we multiply Eq. (12) by the characteristic advective time $\tau=\frac{r_{m}-\sigma_{L J}}{\langle V\rangle}$, where $r_{m} \approx$ $1.122 \sigma_{L J}$ is the distance at which the long range potential reaches its minimum (maximum attraction). As a result we obtain,

$$
E=6 \pi \eta_{0}\left(r_{m}-\sigma_{L J}\right) R\langle V\rangle .
$$

For the cases under scrutiny, both $E$ and $\epsilon_{L J}$ are much larger then $k_{B} T$, hence, the latter can be disregarded. Accordingly, we are left with two energy scales, namely the dissipative energy, $E$ and the strength of long-range interaction $\epsilon_{L J}$. When $\epsilon_{L J} \ll E$ we expect that the local inhomogeneity promoted by the long-range interactions will be suppressed due to the action of the external force and hence the linear response should be recovered. In contrast, when $\epsilon_{L J} \gtrsim E$ we expect the onset of nonlinear response due to the local rearrangements in the colloidal density induced by the long-range interactions. Accordingly, substituting Eq. (13) into Eq. (14) and equating it to $\epsilon_{L J}$ provides an estimate for the onset of pressure for the non-Newtonian response,

$$
\frac{\Delta p^{*}}{L}=2 \frac{\eta_{\mathrm{eff}}(\phi) \epsilon_{L J}}{\pi \eta_{0}\left(r_{m}-\sigma_{L J}\right) R h^{2}} .
$$



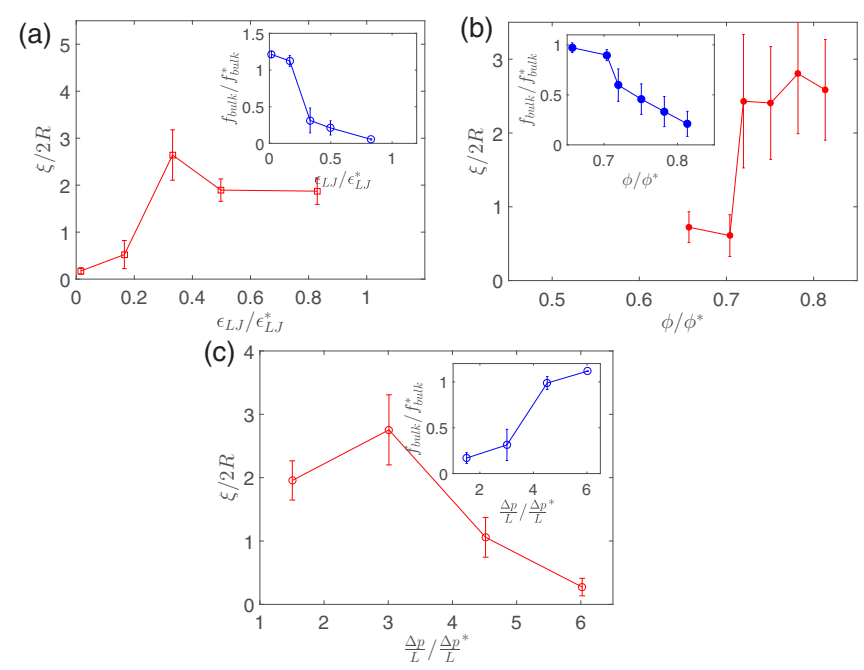

FIG. 7. Values of $\xi$ in unit of $2 R$, and $f_{\text {bulk }}$, used when fitting Eq. (9) and Eq. (10) to the numerical data presented in Fig. 4(a), in Fig. 5(b), in Fig. 5(c). In all cases, the error bars represent confidence interval for the parameters reproducing the data with error $\Delta=\sqrt{\frac{\sum\left(v_{i}-v_{p}\right)^{2}}{n}}$ less than $5 \%$, where $v_{i}$ and $v_{p}$ are the numerical results and the model prediction, respectively. The values of $\epsilon_{L J}^{*}$, $\frac{\Delta p^{*}}{L}$, and $f_{\text {bulk }}^{*}$ used in each case are explained in the text.

Whereas for fixed $\epsilon_{L J}$ and $\frac{\Delta p}{L}$ the critical value of volume fraction reads:

$$
\phi^{*}=\frac{\chi^{\frac{2}{5}}-1}{\chi^{\frac{2}{5}}-c+\chi^{\frac{2}{5}} c}
$$

with

$$
\chi=\frac{\pi\left(r_{m}-\sigma_{L J}\right) R h^{2}}{2 \epsilon_{L J}} \frac{\Delta p}{L} .
$$

While for fixed $\frac{\Delta p}{L}$ and $\phi$ [using Eq. (11)] the critical values of the Lennard-Jones potential:

$$
\epsilon_{L J}^{*}=E=\frac{\pi \eta_{0}\left(r_{m}-\sigma_{L J}\right) R h^{2}}{2 \eta_{\mathrm{eff}}(\phi)} \frac{\Delta p}{L} .
$$

For clarity reasons, Fig. 7 summarizes, the values of the fluidity length $\xi$ in units of $2 R$, and bulk fluidity $f_{\text {bulk }}$, used to fit the nonuniform model [Eq. (9) and Eq. (10)] to the numerical data of Fig. 4, Fig. 5, and Fig. 6.

In particular, Fig. 7(a) corresponds to the data shown in in Fig. 4, i.e., when changing $\epsilon_{L J}$ with constant $\phi=0.52$ and $\frac{\Delta p}{L}=5 \times 10^{-5}$. In this case, the values of $\epsilon_{L J}$ are rescaled by the characteristic energy $\epsilon_{L J}^{*}$ [see Eq. (18)] and $f_{\text {bulk }}$ is normalized by the bulk fluidity calculated using Eq. (11), $f_{\text {bulk }}^{*}=1 / \eta_{\text {eff }}(\phi)$. It is noticeable that introducing the longrange interactions potential leads to the increasing of the fluidity length $\xi$ and the reduction of the bulk fluidity $f_{\text {bulk }}$. Interestingly, we found that there is a threshold value $\epsilon_{L J} \approx$ $0.4 \epsilon_{L J}^{*}\left(\beta \epsilon_{L J} \simeq 500\right)$ above which the fluidity length, $\xi$, is of the order of a few particle diameters whereas for $\epsilon_{L J} \ll$ $0.4 \epsilon_{L J}^{*}$ we have that $\xi$ becomes vanishing small. Moreover, for $\epsilon_{L J}<0.4 \epsilon_{L J}^{*}$ we have $f_{\text {bulk }} / f_{\text {bulk }}^{*} \simeq 1$ whereas for $\epsilon_{L J}>$ $0.4 \epsilon_{L J}^{*}$ the bulk fluidity strongly diminishes [see inset of Fig. 7(a)].
Figure 7(b) shows how the onset of nonuniform rheology depends on the suspension volume fraction, $\phi$, for fixed $\epsilon_{L J}=0.2$ and $\frac{\Delta p}{L}=5 \times 10^{-5}$ (corresponding to Fig. 5). The values of $f_{\text {bulk }}$ are rescaled with $f_{\text {bulk }}^{*}=1 / \eta_{\text {eff }}(\phi)$, where $\phi=0.42$, i.e., the lower explored value. Moreover, the values of volume fraction are rescaled with $\phi^{*}$, which is estimated using Eq. (18). Figure 7(b) shows that high values of $\phi$, which represent the most confined situation, leads to lower values of bulk fluidity $f_{\text {bulk }}$ and larger effective viscosity. Moreover, an abrupt change in the nonuniform fluidity length $\xi$ is detected for an specific value of volume fraction. These results suggest that the onset of the non-Newtonian regime is due to a nontrivial coupling between the long-range interactions among colloids and the confinement. In particular, for $\frac{\Delta p}{L}=$ $5 \times 10^{-5}$ and $\epsilon_{L J}=0.2$, we find non-Newtonian rheology sets for values of $\phi$ above the threshold value $0.75 \phi^{*}$.

Finally, Fig. 7(c) summarizes the results varying the forcing, $\frac{\Delta p}{L}$ (corresponding to Fig. 6). As we pointed out earlier, for weak values of $\frac{\Delta p}{L}$ the system shows a non-Newtonian response, switching to a Newtonian one upon increasing $\frac{\Delta p}{L}$. Interestingly, Fig. 7(c) shows that the onset of the nonNewtonian rheology $\frac{\Delta p}{L} \approx 2 \frac{\Delta p^{*}}{L}$ where we evaluate $\epsilon_{L J}=$ 0.2 and $\phi=0.52$ in the scaling function $\frac{\Delta p^{*}}{L}$ Eq. (15). These results validate the consistency of our analysis.

\section{B. Suspension morphology}

The balance between the energy input and internal dissipation always leads to steady-state regimes, but revealing diverse flow morphologies. Thus, we examine the one-point correlation function $g_{z}(z)$, i.e., the probability that a colloid lies at a given distance $z$ from the wall averaged over the $x-y$ plane. Note that $g_{z}(z)$ is proportional to an average volume fraction profile. Moreover, we quantify the degree of local ordering on the $x-y$ plane, by means of the radial distribution function $g_{x y}(r)$, which accounts for the probability of finding two colloids separated by distance $r=\sqrt{\Delta x^{2}+\Delta y^{2}}$, regions of width $\Delta z=2 R$. Complementarily, we describe the local structural order in the $x-y$ plane, by using the hexatic order parameter $\Psi_{6}$, defined as,

$$
\Psi_{6}=\left|\frac{1}{N} \sum_{j=1}^{N} \varphi_{6}\left(\vec{r}_{j}\right)\right|
$$

with

$$
\varphi_{6}\left(\vec{r}_{j}\right)=\frac{1}{n_{j}} \sum_{k=1}^{n_{j}} \exp \left(i 6 \theta_{j k}\right) .
$$

In the above equation, $\theta_{j k}$ is the angle between the neighbors $k$ of each particle $j$. Specifically, the sum runs over the $n_{j}$ neighbors at a distance $r<1.1 \times D$, where $D=2 R$. Thus, $\Psi_{6}$ is obtained averaging the result of the $N$ particles of the layer.

First, we discuss the case without long-range interaction $\left(\epsilon_{L J}=0\right)$. Figure 8(a) illustrates the correlation function profiles $g_{z}(z)$ obtained for different volume fractions. For comparison, we also show the $g_{z}(z)$ obtained initially, when the colloids are distributed in random positions inside the simulation box. We find that for systems with high volume 

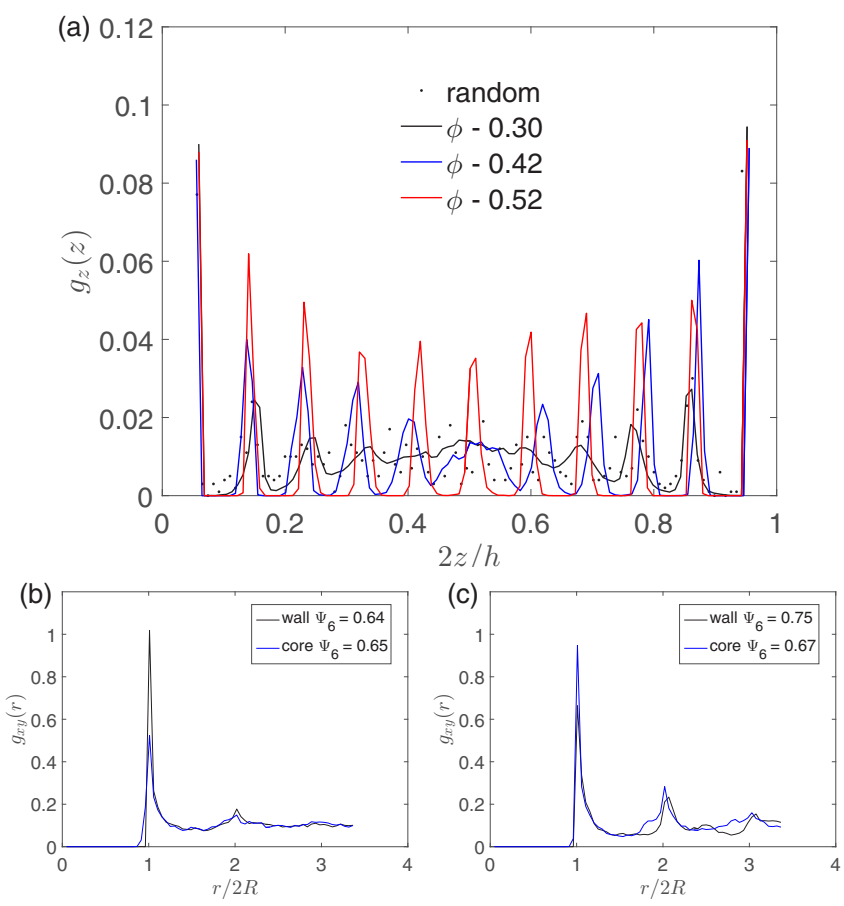

FIG. 8. Correlation functions profiles, obtained in the absence of long-range interactions $\left(\epsilon_{L J}=0\right)$. (a) One-point correlation function $g_{z}(z)$ profiles for $\phi=[0.30 ; 0.34 ; 0.42 ; 0.52]$. Radial distribution function $g_{x y}(r)$ profiles, obtained for (b) $\phi=0.3$ and (c) $\phi=0.52$.

fraction, the profiles of $g_{z}(z)$ show local peaks, which denotes a pronounced layerlike ordering and the strong degree of correlation between colloid movement and the walls. Figure 8(a) also shows that for higher values of the volume fraction the distance among the layers $\Delta h \approx 9.45$ is slightly smaller than $2 R$, which suggests that the colloids are ordered as in a honeycomb lattice. Interestingly, the high of the peaks of $g_{z}(z)$ is not uniform and the peaks close to the walls become larger than those close to the channel axis upon increasing the volume fraction. Moreover, as the volume fraction decreases (by increasing the channel width $W$ with constant number of particles), the amplitude of the peaks of $g_{z}(z)$ reduces, which indicates loss of layering. Thus, at the smallest channel width $(\phi=0.30)$, the layering across the system practically breaks down and the walls no longer induce well-ordered layering in agreement with previously reported numerical $[18,51-53]$ and experimental [10,54-57] studies. Complementarily, in Fig. 8(b) and Fig. 8(c) the degree of local ordering on the $x-y$ plane is studied using radial distribution function $g_{x y}(r)$, for two extreme values of volume fraction. Note that the maximum of the curves are always located at values of $2 R$, which are multiples of the particle diameter, suggesting that a cubic phase is dominant in the horizontal structure. As expected, it is found that the changes in the two-point correlation function correlates with the differences in the hexatic order parameter, $\Psi_{6}$ (see legends in Fig. 8).

In previous studies, this particle layering has been related with an anomalous behavior of the suspension viscosity when changing the volume fraction $[18,52,58]$. However, for $\epsilon_{L J}=0$ we obtain a Newtonian response and a monotonous increase of the viscosity with the volume fraction. Thus, our
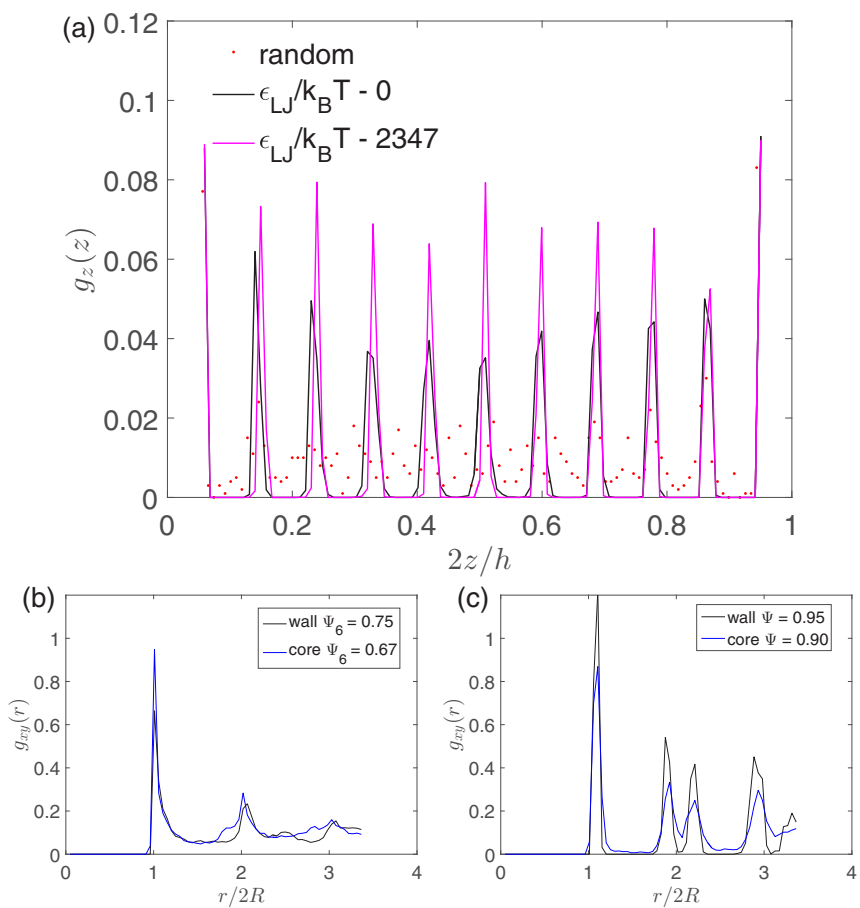

FIG. 9. Correlation functions profiles, obtained varying the longrange interaction potential $\epsilon_{L J}$. (a) One-point correlation functions $g_{z}(z)$. The results correspond to $\frac{\Delta p}{L}=5.0 \times 10^{-5}, \epsilon_{S D}=0.5, \phi=$ 0.52 . Radial distribution function $g_{x y}(r)$ profiles, obtained for two values of $\epsilon_{L J}$, (b) below and (c) above the transition

results suggest that the shear-induced hydrodynamic forces dominate over the long-range wall effects, for the range of explored volume fractions.

Next, in Fig. 9(a), we explore the changes in the morphology of the suspension, which are detected varying the strength of the long-range interaction potential $\epsilon_{L J}$ at fixed volume fraction, $\phi=0.52$, and external force, $\frac{\Delta p}{L}=5 \times 10^{-5}$. In contrast to what we have shown for $\epsilon_{L J}=0$, for very large values of the long-range interaction potential $\epsilon_{L J}$ we find that the wall-induced layering spreads homogeneously across the whole channel, denoting the large-range correlations are enhanced. Complementarily, Fig. 9(b) and Fig. 9(c) illustrate the radial distribution function $g_{x y}(r)$ obtained for two extreme values of long-range interaction potential $\epsilon_{L J}$. Interestingly, while for $\epsilon_{L J}=0$ a cubic phase results dominant in the horizontal structure, for $\epsilon_{L J} / \epsilon^{*} \approx 0.8$ (or equivalently $\epsilon_{L J}=2347 k_{B} T$ ) the position of the maximum indicates that a hexagonal phase is dominant. This change in the $g(r)$ is in agreement with the values of the mean hexatic order parameter $\Psi_{6}$ (see legends in Fig. 9). It is noticeable that the long-range interaction potential induces a significant change in the morphology of the suspension, which correlates with the change in the rheological response (see Fig. 4).

Finally, varying the pressure drop $\frac{\Delta p}{L}$ and keeping $\epsilon_{L J} / \epsilon^{*} \approx 0.8$ (or equivalently $\epsilon_{L J}=2347 k_{B} T$ ) and $\phi=$ 0.52 [see Fig. 10(a)], we obtain that the nature of wallinduced layering is not altered. Note, that the hight of the maximums is the practically the same in the whole channel and for both cases. Moreover, in this case both the radial distribution function $g_{x y}(r)$ and the hexatic order parameter 

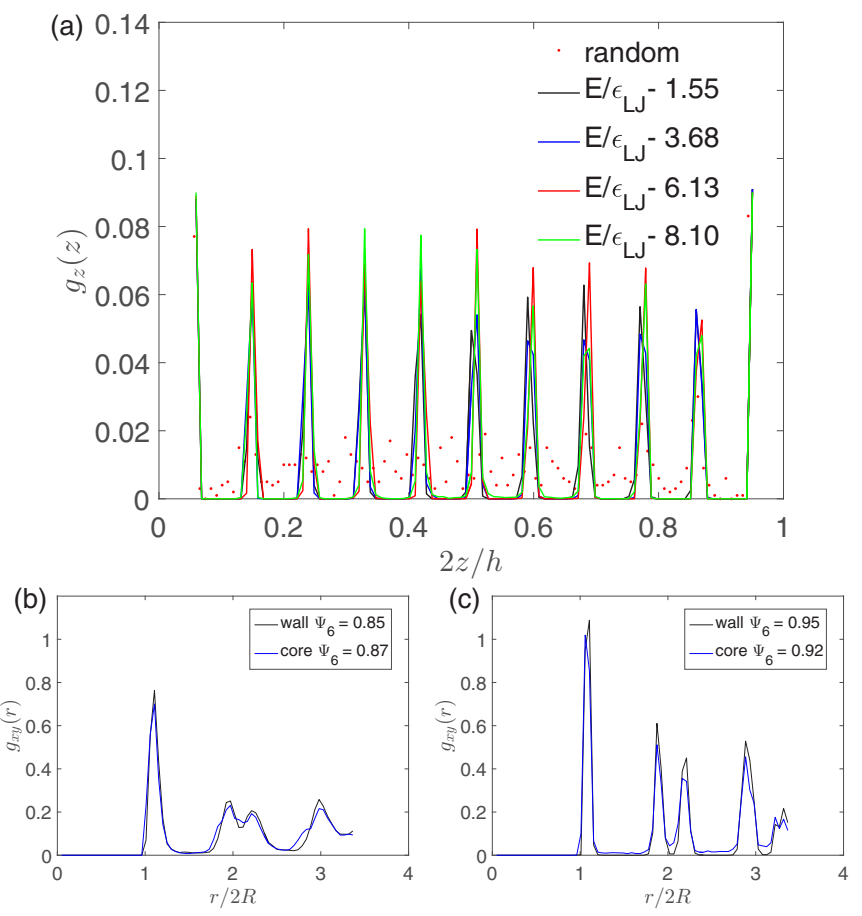

FIG. 10. Correlation functions profiles, obtained varying the pressure drop $\frac{\Delta p}{L}$. The results correspond to $\epsilon_{L J}=0.2, \epsilon_{S D}=0.5$, $\phi=0.52$. (a) One-point correlation function $g_{z}(z)$. Radial distribution function $g_{x y}(r)$ profiles, obtained for two pressure drops $\frac{\Delta p}{L}$ (b) $\frac{\Delta p}{L}=10^{-4}$ and (c) $\frac{\Delta p}{L}=2.5 \times 10^{-5}$, which is below the transition.

presented in Fig. 10(b) and Fig. 10(c), indicates a mild change in the horizontal plane upon increasing $\frac{\Delta p}{L}$. Interestingly, both indicators show a weakening of the hexagonal order upon increasing $\frac{\Delta p}{L}$. This indicates that larger values of $\frac{\Delta p}{L}$ (i.e., larger than those we could attain) may destroy the hexagonal order.

\section{CONCLUSIONS}

We examine numerically in three dimensions, the rheological response of colloidal suspensions when flowing between parallel plates under the action of a constant pressure gradient. When the colloids interact solely via a short-range potential the colloidal suspension displays a Newtonian response, for the studied volume fraction. Moreover, the colloids order in layers parallel to the streamlines. For the highest value of the volume fraction $(\phi=0.52)$, we obtain the distance among layers is smaller that the particle diameter, hence, pinpointing that colloids are organized in a honeycomblike structure. When introducing a long-range Lennard-Jones potential the macroscopic response of the suspensions changes, from Newtonian to non-Newtonian, depending on the interaction strength, the volume fraction and the pressure drop. Exploiting a theoretical framework proposed by Goyon et al. [14,15] we rationalize the simulations data and identify the relevant energy scales involved in this transport process. The latter allows us to propose some scaling relations, which are in good agreement with the numerical data. Finally, we find that the distribution of colloids among layers does not correlate with changes in the macroscopic response. However, the changes in the macroscopic response partially correlates with the type of structure within individual layers. Suspensions characterized by a Newtonian response display a more cubiclike structure of colloids within individual layers, whereas suspensions with non-Newtonian response colloids organize in a more hexagonal-like structure.

\section{ACKNOWLEDGMENTS}

The Spanish MINECO (Projects FIS2017-84631-P) and PIUNA University of Navarra supported this work. The authors thank I. Zuriguel for fruitful discussions and F. Alarcon for his technical support.
[1] P. Coussot, Rheometry of Pastes, Suspensions and Granular Materials-Application in Industry and Environment (Wiley, New York, 2005).

[2] P. Coussot, Rheophysics of pastes: a review of microscopic modelling approaches, Soft Matter 3, 528 (2007).

[3] D. Brogioli, Extracting Renewable Energy from a Salinity Difference Using a Capacitor, Phys. Rev. Lett. 103, 058501 (2009).

[4] J. Guzowski, K. Gizynski, J. Gorecki, and P. Garstecki, Microfluidic platform for reproducible self-assembly of chemically communicating droplet networks with predesigned number and type of the communicating compartments, Lab Chip 16, 764 (2016).

[5] A. M. Forsyth, J. Wan, P. D. Owrutsky, M. Abkarian, and H. A. Stone, Multiscale approach to link red blood cell dynamics, shear viscosity, and atp release, PNAS 108, 10986 (2011).

[6] G. R. Lazaro, A. Hernandez-Machado, and I. Pagonabarraga, Rheology of red blood cells under flow in highly confined microchannels. ii. effect of focusing and confinement, Soft Matter 10, 7207 (2014).
[7] M. Thiébaud, Z. Shen, J. Harting, and C. Misbah, Prediction of Anomalous Blood Viscosity in Confined Shear Flow, Phys. Rev. Lett. 112, 238304 (2014).

[8] M. Brust, O. Aouane, M. Thiébaud, D. Flormann, C. Verdier, L. Kaestner, M. W. Laschke, H. Selmi, A. Benyoussef, T. Podgorski, G. Coupier, C. Misbah, and C. Wagner, The plasma protein fibrinogen stabilizes clusters of red blood cells in microcapillary flows, Sci. Rep 4, 4348 (2014).

[9] P. Malgaretti, I. Pagonabarraga, and J. M. Rubi, Entropic Electrokinetics, Phys. Rev. Lett 113, 128301 (2014).

[10] O. Bunk, A. Diaz, F. Pfeiffer, C. David, C. Padeste, H. Keymeulen, P. R. Willmott, B. D. Patterson, B. Schmitt, D. K. Satapathy, J. F. van der Veen, H. Guo, and G. H. Wegdam, Confinement-induced liquid ordering investigated by x-ray phase retrieval, Phys. Rev. E 75, 021501 (2007).

[11] T. Börzsönyi, B. Szabó, G. Törös, S. Wegner, J. Török, E. Somfai, T. Bien, and R. Stannarius, Orientational Order and Alignment of Elongated Particles Induced by Shear, Phys. Rev. Lett. 108, 228302 (2012). 
[12] S. Wegner, R. Stannarius, A. Boese, G. Rose, B. Szabo, E. Somfai, and T. Borzsonyi, Effects of grain shape on packing and dilatancy of sheared granular materials, Soft Matter 10, 5157 (2014).

[13] J.-B. Salmon, L. Bécu, S. Manneville, and A. Colin, Towards local rheology of emulsions under couette flow using dynamic light scattering, Eur. Phys. J E Soft 10, 209 (2003).

[14] J. Goyon, A. Colin, G. Ovarlez, A. Ajdari, and L. Bocquet, Spatial cooperativity in soft glassy flows, Nature (London) $\mathbf{4 5 4}$, 84 (2008).

[15] J. Goyon, A. Colin, and L. Bocquet, How does a soft glassy material flow: Finite size effects, non local rheology, and flow cooperativity, Soft Matter 6, 2668 (2010).

[16] S. Manneville, L. Bécu, and A. Colin, High-frequency ultrasonic speckle velocimetry in sheared complex fluids, Eur. Phys. J. Appl. Phys. 28, 361 (2004).

[17] S. Mueller, E. W. Llewellin, and H. M. Mader, The rheology of suspensions of solid particles, Proc. R. Soc. A 466, 1201 (2010).

[18] S. Gallier, E. Lemaire, L. Lobry, and F. Peters, Effect of confinement in wall-bounded non-colloidal suspensions, J. Fluid Mech. 799, 100 (2016).

[19] V. Doyeux, S. Priem, L. Jibuti, A. Farutin, M. Ismail, and P. Peyla, Effective viscosity of two-dimensional suspensions: Confinement effects, Phys. Rev. Fluids 1, 043301 (2016).

[20] M. Foglino, A. N. Morozov, O. Henrich, and D. Marenduzzo, Flow of Deformable Droplets: Discontinuous Shear Thinning and Velocity Oscillations, Phys. Rev. Lett. 119, 208002 (2017).

[21] M. E. Rosti, L. Brandt, and D. Mitra, Rheology of suspensions of viscoelastic spheres: Deformability as an effective volume fraction, Phys. Rev. Fluids 3, 012301 (2018).

[22] K. Suzuki and H. Hayakawa, arXiv:1711.08855.

[23] K. Nichol, A. Zanin, R. Bastien, E. Wandersman, and M. van Hecke, Flow-Induced Agitations Create a Granular Fluid, Phys. Rev. Lett. 104, 078302 (2010).

[24] K. A. Reddy, Y. Forterre, and O. Pouliquen, Evidence of Mechanically Activated Processes in Slow Granular Flows, Phys. Rev. Lett. 106, 108301 (2011).

[25] M. Bouzid, M. Trulsson, P. Claudin, E. Clément, and B. Andreotti, Nonlocal Rheology of Granular Flows Across Yield Conditions, Phys. Rev. Lett. 111, 238301 (2013).

[26] D. L. Henann and K. Kamrin, Continuum Modeling of Secondary Rheology in Dense Granular Materials, Phys. Rev. Lett. 113, 178001 (2014).

[27] D. Chen, K. W. Desmond, and E. R. Weeks, Experimental observation of local rearrangements in dense quasi-twodimensional emulsion flow, Phys. Rev. E 91, 062306 (2015).

[28] M. Bouzid, A. Izzet, M. Trulsson, E. Clément, P. Claudin, and B. Andreotti, Non-local rheology in dense granular flows, Eur. Phys. J E Soft 38, 125 (2015).

[29] M. Bouzid, M. Trulsson, A. Izzet, A. F. de Coulomb, P. Claudin, E. Clément, and B. Andreotti, Non-local rheology of dense granular flows, EPJ Web Conf. 140, 11013 (2017).

[30] T. Gueudre, J. Lin, A. Rosso, and M. Wyart, Scaling description of non-local rheology, Soft Matter 13, 3794 (2017).

[31] B. Géraud, L. Jørgensen, C. Ybert, H. Delanoë-Ayari, and C. Barentin, Structural and cooperative length scales in polymer gels, Eur. Phys. J E Soft 40, 5 (2017).

[32] P. Jop, V. Mansard, P. Chaudhuri, L. Bocquet, and A. Colin, Microscale Rheology of a Soft Glassy Material Close to Yielding, Phys. Rev. Lett. 108, 148301 (2012).
[33] M. Sbragaglia, R. Benzi, M. Bernaschi, and S. Succi, The emergence of supramolecular forces from lattice kinetic models of non-ideal fluids: applications to the rheology of soft glassy materials, Soft Matter 8, 10773 (2012).

[34] S. Succi, The lattice Boltzmann equation: for fluid dynamics and beyond (Oxford University Press, Oxford, 2001).

[35] M. E. Cates, K. Stratford, R. Adhikari, P. Stansell, J. C. Desplat, I. Pagonabarraga, and A. J. Wagner, Simulating colloid hydrodynamics with lattice boltzmann methods, J. Phys.: Condens. Matter 16, S3903 (2004).

[36] J. Harting, J. Chin, M. Venturoli, and P. V. Coveney, Large-scale lattice boltzmann simulations of complex fluids: Advances through the advent of computational grids, Philos. Trans. Royal Soc. A 363, 1895 (2005).

[37] J. Harting, C. Kunert, and J. Hyväluoma, Lattice boltzmann simulations in microfluidics: Probing the no-slip boundary condition in hydrophobic, rough, and surface nanobubble laden microchannels, Microfluid Nanofluid 8, 1 (2009).

[38] N. Rivas, S. Frijters, I. Pagonabarraga, and J. Harting, Mesoscopic electrohydrodynamic simulations of binary colloidal suspensions, J. Chem. Phys. 148, 144101 (2018).

[39] E. S. Asmolov, A. L. Dubov, T. V. Nizkaya, J. Harting, and O. I. Vinogradova, Inertial focusing of finite-size particles in microchannels, J. Fluid Mech. 840, 613 (2018).

[40] J. C. Desplat, I. Pagonabarraga, and P. Bladon, Ludwig: A parallel lattice-boltzmann code for complex fluids, Comput. Phys. Commun 134, 273 (2001).

[41] R. Adhikari, K. Stratford, M. E. Cates, and A. J. Wagner, Fluctuating lattice boltzmann, Europhys. Lett. 71, 473 (2005).

[42] S. P. Thampi, I. Pagonabarraga, and R. Adhikari, Latticeboltzmann-langevin simulations of binary mixtures, Phys. Rev. E 84, 046709 (2011).

[43] G. Kähler, F. Bonelli, G. Gonnella, and A. Lamura, Cavitation inception of a van der waals fluid at a sack-wall obstacle, Phys. Fluids 27, 123307 (2015).

[44] G. Bryant, S. R. Williams, L. Qian, I. K. Snook, E. Perez, and F. Pincet, How hard is a colloidal "hard-sphere" interaction? Phys. Rev. E 66, 060501 (2002).

[45] N.-Q. Nguyen and A. J. C. Ladd, Lubrication corrections for lattice-boltzmann simulations of particle suspensions, Phys. Rev. E 66, 046708 (2002).

[46] M. J. Fuerstman, A. Lai, M. E. Thurlow, S. S. Shevkoplyas, H. A. Stone, and G. M. Whitesides, The pressure drop along rectangular microchannels containing bubbles, Lab Chip 7, 1479 (2007).

[47] G. K. Batchelor and J. T. Green, Brownian diffusion of particles with hydrodynamic interaction, J. Fluid. Mech 56, 401 (1972).

[48] C. I. Mendoza and I. Santamaría-Holek, The rheology of hard sphere suspensions at arbitrary volume fractions: An improved differential viscosity model, J. Chem. Phys. 130, 044904 (2009).

[49] D. Quemada, Rheology of concentrated disperse systems and minimum energy dissipation principle, Rheol. Acta 16, 82 (1977).

[50] I. M. Krieger and T. J. Dougherty, A mechanism for nonnewtonian flow in suspensions of rigid spheres, J. Rheol. 3, 137 (1959). 
[51] A. Komnik, J. Harting, and H. J. Herrmann, Transport phenomena and structuring in shear flow of suspensions near solid walls, J. Stat. Mech. (2004) P12003.

[52] K. Yeo and M. R. Maxey, Ordering transition of non-brownian suspensions in confined steady shear flow, Phys. Rev. E 81, 051502 (2010).

[53] R. Hartkamp, A. Ghosh, T. Weinhart, and S. Luding, A study of the anisotropy of stress in a fluid confined in a nanochannel, J. Chem. Phys. 137, 044711 (2012).

[54] X. Cheng, J. H. McCoy, J. N. Israelachvili, and I. Cohen, Imaging the microscopic structure of shear thinning and thickening colloidal suspensions, Science 333, 1276 (2011).
[55] M. Zurita-Gotor, J. Bławzdziewicz, and E. Wajnryb, Layering Instability in a Confined Suspension Flow, Phys. Rev. Lett. 108, 068301 (2012).

[56] B. Snook, J. E. Butler, and E. Guazzelli, Dynamics of shearinduced migration of spherical particles in oscillatory pipe flow, J. Fluid Mech. 786, 128 (2016).

[57] S. Pieper and H. J. Schmid, Layer-formation of non-colloidal suspensions in a parallel plate rheometer under steady shear, J. Nonnewton Fluid Mech 234, 1 (2016).

[58] S. D. Kulkarni and J. F. Morris, Ordering transition and structural evolution under shear in brownian suspensions, J. Rheol. 53, 417 (2009). 